Moroccan J. of Pure and Appl. Anal. (MJPAA)

Volume 6(2), 2020, Pages 210-217

ISSN: Online 2351-8227 - Print 2605-6364

DOI: 10.2478/mjpaa-2020-0016

\title{
On some properties of the conformable fractional derivative
}

\author{
Radouane Azennar ${ }^{1}$ and Driss Mentagui ${ }^{2}$
}

Aвstract. In this paper, we prove that the intermediate value theorem remains true for the conformable fractional derivative and we prove some useful results using the definition of conformable fractional derivative given in R. Khalil, M. Al Horani, A. Yousef, M. Sababhehb [4].

Mathematics Subject Classification (2010). 26A33, 26A24.

Key words and phrases. Darboux's theorem, the intermediate value theorem, conformable fractional derivative.

\section{Introduction}

Fractional calculus is one of the most powerful mathematical tools in the recent decades to model real world problems in many fields of science, technology, and engineering. History of fractional calculus (FC) goes back to the seventeenth century. In 1695, the derivative of order $\alpha=\frac{1}{2}$ was described by Leibnitz. Since then, the new theory turned out to be very attractive to mathematicians as well as biologists, chemists, economists, engineers, and physicists. Several books were written on the theories and developments of fractional calculus $[6,8,9,12]$.

Fractional calculus was introduced in one of Niels Henrik Abel's early papers (see for instance[1]) where all the elements can be found: the idea of fractional-order integration and differentiation, the mutually inverse relationship between them, the understanding that fractional-order differentiation and integration can be considered as the same generalized operation, and even

Received 20 May 2020 - Accepted: 29 July 2020.

(C) This article is published with open access by Sidi Mohamed Ben Abdallah University.

The Author(s): ${ }^{1,2}$ Department of Mathematics, Faculty of Sciences, Ibn Tofaïl University, B.P. 133, Kenitra, 14000, Morocco e-mail: ${ }^{1}$ azennar_pf@hotmail.com (Corresponding Author)

e-mail: ${ }^{2}$ dri_mentagui@yahoo.fr . 
the unified notation for differentiation and integration of arbitrary real order [10]. Independently, the foundations of the subject were laid by Liouville in a paper from 1832. The autodidact Oliver Heaviside introduced the practical use of fractional differential operators in electrical transmission line analysis circa 1890 (see [11]). The theory and applications of fractional calculus expanded greatly over the 19th and 20th centuries, and numerous contributors have given definitions for fractional derivatives and integrals. The fractional derivatives are defined by a fractional integral (see for instance $[6,8,9,12])$. There are several known forms of the fractional integrals of which two have been studied extensively for their applications (see for example $[2,3,5,6,8,9,12])$. For $\alpha \in[n-1, n[$, and a function $f$, The first $\alpha$-derivative is the Riemann-Liouville definition given as follows:

$$
D_{a}^{\alpha}(f)(t)=\frac{1}{\Gamma(n-\alpha)} \frac{d^{n}}{d t^{n}} \int_{a}^{t} \frac{f(x)}{(t-x)^{\alpha-n+1}} d x
$$

The second one is the Caputo definition given by:

$$
D_{a}^{\alpha}(f)(t)=\frac{1}{\Gamma(n-\alpha)} \int_{a}^{t} \frac{f^{(n)}(x)}{(t-x)^{\alpha-n+1}} d x .
$$

\section{Notations and preliminaries}

In this section, we recall some basic results which will be used later For more details, we refer the reader to $[4,7]$ and the references therein.

Given a function $f:[0,+\infty[\longrightarrow \mathbb{R}$, Then the conformable fractional $\alpha$-derivative of $f$ is defined by:

$$
\left.T_{\alpha}(f)(t)=\lim _{\varepsilon \rightarrow 0} \frac{f\left(t+\varepsilon t^{1-\alpha}\right)-f(t)}{\varepsilon} \text { for all } t>0, \text { and } \alpha \in\right] 0,1[.
$$

If $f$ is $\alpha$-differentiable on some $(0, a), a>0$, and $\lim _{t \rightarrow 0^{+}} f^{(\alpha)}(t)$ exists, then define

$$
f^{(\alpha)}(0)=\lim _{t \rightarrow 0^{+}} f^{(\alpha)}(t)
$$

In [?], The Riemann-Liouville's (RL) fractional integral operator of order $\alpha \in[n-1, n[$ for a function $f \in L^{1}([a, b])$ is given as follows:

$$
I^{a} w(x)=\frac{1}{\Gamma(n-\alpha)} \int_{a}^{x} w(\tau)(x-\tau)^{n-\alpha-1} d \tau .
$$

Definition 2.1. A function $f:[a, b] \rightarrow \mathbb{R}$ is said to satify the intermediate value property on $[a, b]$, if for every $\lambda$ between $f(a)$ and $f(b)$, there exists $c \in(a, b)$ such that $f(c)=\lambda$.

If $f$ is continuous then it satisfies the intermediate value proprty.

The statement of the Darboux's theorem follows here.

Theorem 2.1. (Darboux's Theorem). If $f$ is differentiable on $[a, b]$ and if $\lambda$ is a real number between $f^{\prime}(a)$ and $f^{\prime}(b)$, then there is at least one point $c \in(a, b)$ such that $f^{\prime}(c)=\lambda$.

In other words, the image $f^{\prime}(I)$ of an interval $I \subset \mathbb{R}$ by $f^{\prime}$ is an interval $\mathbb{R}$. 
Theorem 2.2. ([4, Theorem 2.3]). Let $a>0$ and $f:[a, b] \rightarrow \mathbb{R}$ be a given function that satisfies:

(1) $f$ is continuous on $[a, b]$,

(2) $f$ is $\alpha$-differentiable for some $\alpha \in] 0,1$,

(3) $f(a)=f(b)$.

Then, there exists $c \in] a, b\left[\right.$ such that $f^{(\alpha)}(c)=0$.

Theorem 2.3. ([4, Theorem 2.4]). Let $a>0$ and $f:[a, b] \rightarrow \mathbb{R}$ be a given function satisfies the following assertions:

(1) $f$ is continuous on $[a, b]$,

(2) $f$ is $\alpha$-differentiable for some $\alpha \in] 0,1[$.

Then, there exists $c \in] a, b\left[\right.$, such that $f^{(\alpha)}(c)=\frac{f(b)-f(a)}{\frac{1}{\alpha} b^{\alpha}-\frac{1}{\alpha} \alpha^{\alpha}}$.

The objective of this paper is to prove that the intermediate value theorem remains true if replacing the integer derivative $f^{\prime}$ by the conformable fractional one $f^{(\alpha)}$. We establish also some useful results. Moreover, we give some examples which illustrate the obtained results.

\section{Main results}

In the present section, we will state and prove main results. We start by The following results which generalize the Darboux's theorem, and the previous results in Theorem 2.1 is a particular case of which $\alpha=1$.

Theorem 3.1. Let $a>0$ and $f:[a, b] \rightarrow \mathbb{R}$ is a function $\alpha$-differentiable for some $\alpha \in] 0,1[$ on $[a, b]$ and if $\lambda$ is a number between $f^{(\alpha)}(a)$ and $f^{(\alpha)}(b)$, then there is at least one point $\left.c \in\right] a, b[$ such that $f^{(\alpha)}(c)=\lambda$.

Remark 3.1. $f^{(\alpha)}$ is not necessarily continuous on $[a, b]$.

Proof. First, let $t \in[a, b]$, as $f$ is $\alpha$-differentiable in $t$ and we pose $h=\varepsilon t^{1-\alpha}$ we have :

$$
\begin{aligned}
T_{\alpha}(f)(t):=\lim _{\varepsilon \rightarrow 0} \frac{f\left(t+\varepsilon t^{1-\alpha}\right)-f(t)}{\varepsilon} & =\lim _{h \rightarrow 0} \frac{f(t+h)-f(t)}{h t^{\alpha-1}} \\
& =f^{(\alpha)}(t) .
\end{aligned}
$$

So, we have

$$
\begin{aligned}
\lim _{h \rightarrow 0} \frac{f(t+h)-f(t)}{\frac{(t+h)^{\alpha}}{\alpha}-\frac{t^{\alpha}}{\alpha}} & =\lim _{h \rightarrow 0} \frac{f(t+h)-f(t)}{h t^{\alpha-1}} \times \frac{h t^{\alpha-1}}{\frac{(t+h)^{\alpha}}{\alpha}-\frac{t^{\alpha}}{\alpha}} \\
& =f^{(\alpha)}(t) \times 1 \\
& =f^{(\alpha)}(t) .
\end{aligned}
$$

Since

$$
\lim _{h \rightarrow 0} \frac{h t^{\alpha-1}}{\frac{(t+h)^{\alpha}}{\alpha}-\frac{t^{\alpha}}{\alpha}}=\lim _{h \rightarrow 0} \frac{1}{\frac{(t+h)^{\alpha}-\frac{t^{\alpha}}{\alpha}}{h t^{\alpha-1}}}
$$




$$
\begin{aligned}
& =\frac{1}{T_{\alpha}\left(\frac{1}{\alpha} t^{\alpha}\right)} \\
& =1 .
\end{aligned}
$$

and $T_{\alpha}\left(\frac{1}{\alpha} t^{\alpha}\right)=1$. Now, we suppose $f^{(\alpha)}(a)<f^{(\alpha)}(b)$, let $\left.\lambda \in\right] f^{(\alpha)}(a), f^{(\alpha)}(b)[$. We set $\varepsilon=$ $\min \left(\lambda-f^{(\alpha)}(a), f^{(\alpha)}(b)-\lambda\right)$. By definition of the limit and the $\alpha$-differentiability on the right in $a$, there is $\beta_{1}>0$ such that for all $h$ in $\left.] 0, \beta_{1}\right]$ :

$$
\frac{f(a+h)-f(a)}{\frac{(a+h)^{\alpha}}{\alpha}-\frac{a^{\alpha}}{\alpha}}<f^{(\alpha)}(a)+\varepsilon \leq \lambda .
$$

Likewise, there is $\beta_{2}>0$ such that for all $h$ in $] 0, \beta_{2}$ ]

$$
\frac{f(b+h)-f(b)}{\frac{(b+h)^{\alpha}}{\alpha}-\frac{b^{\alpha}}{\alpha}}>f^{(\alpha)}(b)-\varepsilon \geq \lambda .
$$

For $\beta=\min \left(\beta_{1}, \beta_{2}\right)$, we have

$$
\frac{f(a+h)-f(a)}{\frac{(a+h)^{\alpha}}{\alpha}-\frac{a^{\alpha}}{\alpha}}<\lambda<\frac{f(b+h)-f(b)}{\frac{(b+h)^{\alpha}}{\alpha}-\frac{b^{\alpha}}{\alpha}} .
$$

We fix $h$ in $] 0, \beta[$ and we pose

$$
\Phi(x)=\frac{f(x+h)-f(x)}{\frac{(x+h)^{\alpha}}{\alpha}-\frac{x^{\alpha}}{\alpha}} .
$$

$\Phi$ is continuous and $\lambda \in] \Phi(a), \Phi(b)$, so by the intermediate value theorem, there is $y \in[a, b]$ such that $\Phi(y)=\lambda$.

Now, by applying the Theorem 2.3 in the interval $[y, y+h]$, there is $c \in[y, y+h]$ such that :

$$
\begin{aligned}
f^{(\alpha)}(c) & =\frac{f(y+h)-f(y)}{\frac{(y+h)^{\alpha}}{\alpha}-\frac{y^{\alpha}}{\alpha}} \\
& =\Phi(y) \\
& =\lambda .
\end{aligned}
$$

Finally, $\lambda \in f^{(\alpha)}([a, b])$.

\section{Example 3.1.}

on the interval $[1,2]$, we consider the mapping $f$ defined by:

$$
f(x)= \begin{cases}(x-1)^{2} \sin \left(\frac{1}{(x-1)^{2}}\right) & \text { for } x \neq 1, \\ 0 & \text { for } x=1\end{cases}
$$

For some $\alpha \in] 0,1[$ and for all $x \neq 1$, we have by $[4$, Theorem 2.2]

$$
f^{(\alpha)}(x)=x^{1-\alpha}\left(2(x-1) \sin \left(\frac{1}{(x-1)^{2}}\right)-\frac{2}{(x-1)} \cos \left(\frac{1}{(x-1)^{2}}\right)\right)
$$


For example, for $\alpha=\frac{1}{2}$, we would have :

$$
f^{\left(\frac{1}{2}\right)}(x)=\sqrt{x}\left(2(x-1) \sin \left(\frac{1}{(x-1)^{2}}\right)-\frac{2}{(x-1)} \cos \left(\frac{1}{(x-1)^{2}}\right)\right)
$$

notice that $f$ is $\alpha$-differentiable on $] 1,2]$ and for $x=1$ we get

$$
\left|\frac{f(1+h)-f(1)}{h 1^{\alpha-1}}\right| \leq h\left|\sin \left(1 / h^{2}\right)\right| \leq h .
$$

Hence, $f$ is $\alpha$-differentiable on 1 and $f^{(\alpha)}(1)=0$, which implies that $f$ is $\alpha$-differentiable on $[1,2]$. It is easy to see that $f^{\left(\frac{1}{2}\right)}$ is not continuous in 1 , just take the the sequence $\left(U_{n}\right)_{n \geq 0}$ defined by $U_{n}=$ $\frac{1}{\sqrt{2 n \pi}}+1$ which converges to 1 but $f^{\left(\frac{1}{2}\right)}\left(U_{n}\right)$ does not converge to 1 .

So, $f^{\left(\frac{1}{2}\right)}(1)=0$ and $f^{\left(\frac{1}{2}\right)}(2) \approx 0.85$.

If $\lambda$ is between $f^{\left(\frac{1}{2}\right)}(1)$ and $f^{\left(\frac{1}{2}\right)}(2)$. Then the equation $f^{\left(\frac{1}{2}\right)}(x)=\lambda$ has at least one solution $c$ in $[1,2]$.

Corollary 3.1. Let $a>0$ and $f:[a, b] \rightarrow \mathbb{R}$ is a function $\alpha$-differentiable for some $\alpha \in] 0,1[$ on $[a, b]$ and if $\lambda$ is a number between $f^{(\alpha)}(a)$ and $f^{(\alpha)}(b)$, then The equation $f^{(\alpha)}(x)=\lambda$ has at least one solution $c \in] a, b[$.

Corollary 3.2. Let $a>0$ and $f:[a, b] \rightarrow \mathbb{R}$ is a function $\alpha$-differentiable for some $\alpha \in] 0,1[$ on $[a, b]$ and if $\lambda$ is a number between $f^{(\alpha)}(a)$ and $f^{(\alpha)}(b)$. If moreover $f^{\alpha}$ is strictly monotonic on $[a, b]$. Then, the equation $f^{(\alpha)}(x)=\lambda$ has only one solution $\left.c \in\right] a, b[$.

Example 3.2. on the interval $\left[\frac{\pi}{4}, \frac{\pi}{3}\right]$, we consider the mapping $f$ defined by:

$$
f(x)=\sin (x) \text {. }
$$

For some $\alpha \in] 0,1[$, by the $[4$, Theorem 2.2], we have

$$
f^{(\alpha)}(x)=x^{1-\alpha} \cos (x)
$$

So, we have for $\alpha=\frac{1}{\pi}, f^{\left(\frac{1}{\pi}\right)}\left(\frac{\pi}{4}\right) \approx 0.599$ and $f^{\left(\frac{1}{\pi}\right)}\left(\frac{\pi}{3}\right) \approx 0.515$.

Since $f^{\left(\frac{1}{\pi}\right)}\left(\frac{\pi}{3}\right)<0.54<f^{\left(\frac{1}{\pi}\right)}\left(\frac{\pi}{4}\right)$ and $f^{\left(\frac{1}{\pi}\right)}$ is decreasing on $\left[\frac{\pi}{4}, \frac{\pi}{3}\right]$.

By approximation, the equation $f^{\left(\frac{1}{\pi}\right)}(x)=0.54$ has one and only one solution $c \approx 1.001$ in $\left[\frac{\pi}{4}, \frac{\pi}{3}\right]$.

Definition 3.1. Let $f$ be an mapping defined on an interval I. We say that $f$ is a strictly $\alpha$-contractive for some $\alpha \in] 0,1[$ if:

$$
\exists K \in\left[0,1\left[, \forall x, y \in I,|f(x)-f(y)| \leq K\left|x^{\alpha}-y^{\alpha}\right| .\right.\right.
$$

Theorem 3.2. Let $a>0$ and $T:[a, b] \rightarrow \mathbb{R}$ be a given function that satisfies :

(1) $T$ is continuous on $[a, b]$.

(2) $T$ is $\alpha$-differentiable for some $\alpha \in] 0,1[$.

(3) $\left.\max _{x \in[a, b]} \mid T^{(} \alpha\right)(x) \mid=K<\alpha$

Then, $T$ is a strictly $\alpha$-contractive. 
Proof. Let $x$ and $y$ be two reals of the interval $[a, b]$, by [4, Theorem 2.4], there exists $c \in] x, y[$, such that $T^{(\alpha)}(c)=\frac{T(y)-T(x)}{\frac{1}{\alpha} y^{\alpha}-\frac{1}{\alpha} x^{\alpha}}$.

Therefore,

$$
\begin{aligned}
|T(x)-T(y)| & =\left|T^{(\alpha)}(c)\left(\frac{1}{\alpha} y^{\alpha}-\frac{1}{\alpha} x^{\alpha}\right)\right| \\
& =\left|\frac{T^{(\alpha)}(c)}{\alpha}\right|\left|y^{\alpha}-x^{\alpha}\right| \\
& \leq \frac{K}{\alpha}\left|y^{\alpha}-x^{\alpha}\right| \\
& \leq K^{\prime}\left|y^{\alpha}-x^{\alpha}\right| .
\end{aligned}
$$

Then, $T$ is a strictly $\alpha$-contractive withe $K^{\prime}=\frac{K}{\alpha}<1$

\section{Example 3.3.}

(1) On the interval $\left[\frac{1}{10}, 1\right]$, we consider the mapping $T$ defined by:

$$
T(x)=\cos (x)
$$

For some $\alpha \in] 0,1[$, by [4, Theorem 2.2], we have

$$
T^{(\alpha)}(x)=-x^{1-\alpha} \sin (x) \text { and } \max _{x \in\left[\frac{1}{10}, 1\right]}\left|T^{(\alpha)}(x)\right|=K<\alpha,
$$

Thus, for $\alpha=\frac{e}{\pi}$, we have

$$
\max _{x \in\left[\frac{1}{10}, 1\right]}\left|T^{\left(\frac{e}{\pi}\right)}(x)\right|=0.841<\frac{e}{\pi} .
$$

Then, $T$ is a strictly $\frac{e}{\pi}$-contractive.

(2) On the interval $[1,2]$, we consider the mapping $T$ defined by:

$$
T(x)=\frac{20}{x^{2}+2 x+10} .
$$

For some $\alpha \in] 0,1$, by [4, Theorem 2.2], we obtain

$$
T^{(\alpha)}(x)=\frac{-40(x+1) x^{1-\alpha}}{\left(x^{2}+2 x+10\right)^{2}} \text { and } \max _{x \in[1,2]}\left|T^{(\alpha)}(x)\right|=K<\alpha,
$$

So, for $\alpha=\frac{10}{11}$, we have

$$
\max _{x \in[1,2]}\left|T^{(\alpha)}(x)\right|=0.473<\alpha .
$$

Then, $T$ is a strictly $\frac{10}{11}$-contractive.

The following theorem is a generalization of Theorem 2.3.

Theorem 3.3. Let $a>0$ and $f, g:[a, b] \rightarrow \mathbb{R}$ be two given functions such that :

(1) $f$ and $g$ are continuous on $[a, b]$, 
(2) $f$ and $g$ are $\alpha$-differentiable for some $\alpha \in] 0,1[$.

Then, there exists $c \in] a, b[$, such that :

$$
g^{(\alpha)}(c)(f(b)-f(a))=f^{(\alpha)}(c)(g(b)-g(a)) .
$$

Proof. Consider the function $h$ defined by

$$
h(x)=f(x)(g(b)-g(a))-g(x)(f(b)-f(a))
$$

then, the function $h$ satisfies the conditions of Theorem 2.2. Hence, there exists $c \in] a, b[$ such that

$$
h^{(\alpha)}(c)=0 \text { where } h^{(\alpha)}(c)=\lim _{\varepsilon \rightarrow 0} \frac{h\left(c+\varepsilon c^{1-\alpha}\right)-h(c)}{\varepsilon} .
$$

Therefore, there exists $c \in] a, b[$ such that

$$
g^{(\alpha)}(c)(f(b)-f(a))=f^{(\alpha)}(c)(g(b)-g(a)) .
$$

The following results generalize the L'Hopital's Rule will be a particular case of which $\alpha=1$.

Theorem 3.4. Let $a>0$ and $f, g:[a, b] \rightarrow \mathbb{R}$ be two given functions such that

(1) $f$ and $g$ are continuous on $[a, b]$,

(2) $f$ and $g$ are $\alpha$-differentiable for some $\alpha \in] 0,1[$,

(3) $g^{(\alpha)}(x)$ is never zero on $] a, b\left[\right.$, and that $\lim _{x \rightarrow a^{+}} \frac{f^{(\alpha)}(x)}{g^{(\alpha)}(x)}=l$.

Then

$$
\lim _{x \rightarrow a^{+}} \frac{f(x)-f(a)}{g(x)-g(a)}=\lim _{x \rightarrow a^{+}} \frac{f^{(\alpha)}(x)}{g^{(\alpha)}(x)}=l .
$$

Proof. By th hypotheses (1) and (2), we can apply Theorem 3.3, for all $x \in[a, b]$, there exists $\left.c_{x} \in\right] a, x[$ such that

$$
g^{(\alpha)}\left(c_{x}\right)(f(x)-f(a))=f^{(\alpha)}\left(c_{x}\right)(g(x)-g(a))
$$

Consequently,

$$
\frac{f(x)-f(a)}{g(x)-g(a)}=\frac{f^{(\alpha)}\left(c_{x}\right)}{g^{(\alpha)}\left(c_{x}\right)}
$$

It is clear that if $x \rightarrow a$, so $c_{x} \rightarrow a$, and thus, by (3) we get

$$
\frac{f^{(\alpha)}\left(c_{x}\right)}{g^{(\alpha)}\left(c_{x}\right)} \rightarrow l
$$

Finally, we have that :

$$
\lim _{x \rightarrow a^{+}} \frac{f(x)-f(a)}{g(x)-g(a)}=\lim _{x \rightarrow a^{+}} \frac{f^{(\alpha)}(x)}{g^{(\alpha)}(x)}=l
$$


Example 3.4. Let's calculate $\lim _{x \rightarrow 0} \frac{1-\cos (x)}{x^{2}}$.

We set that : $f(x)=1-\cos (x)$ and $g(x)=x^{2}$.

We are exactly within conditions for applying the previous result.

$$
\begin{aligned}
\lim _{x \rightarrow 0} \frac{f(x)}{g(x)} & =\lim _{x \rightarrow 0} \frac{f^{(\alpha)}(x)}{g^{(\alpha)}(x)} \\
& =\lim _{x \rightarrow 0} \frac{(1-\cos x)^{(\alpha)}}{\left(x^{2}\right)^{(\alpha)}} \\
& =\lim _{x \rightarrow 0} \frac{x^{1-\alpha} \sin (x)}{2 x^{2-\alpha}} \\
& =\lim _{x \rightarrow 0} \frac{1}{2} \frac{\sin (x)}{x} \\
& =\frac{1}{2} .
\end{aligned}
$$

\section{References}

[1] N. H. Abel, Solution of a couple of problems by means of definite integrals. Magazin for Naturvidenskaberne. Kristiania (Oslo), (1823), 5568.

[2] P.L. Butzer, A.A. Kilbas, J.J. Trujillo, Compositions of Hadamard-type fractional integration operators and the semigroup property, Journal of Mathematical Analysis and Applications 269 (2002) 387400.

[3] P.L. Butzer, A.A. Kilbas, J.J. Trujillo, Fractional calculus in the Mellin setting and Hadamard-type fractional integrals, Journal of Mathematical Analysis and Applications 269 (2002) 127.

[4] R. Khalil, M. Al Horani, A. Yousef , M. Sababhehb, A new definition of fractional derivative.Journal of Computational and Applied Mathematics 264 (2014) 6570.

[5] A. A. Kilbas, Hadamard-type fractional calculus, Journal of Korean Mathematical Society 38 (6) (2001) 11911204.

[6] A.A. Kilbas, H.M. Srivastava, J.J. Trujillo, Theory and Applications of Fractional Differential Equations, Elsevier B.V., Amsterdam, Netherlands, 2006.

[7] O. S. Iyiola and E R. Nwaeze, Some New Results on the New Conformable Fractional Calculus with Application Using DAlambert Approach. Progr. Fract. Differ. Appl. 2, No. 2, 115-122 (2016)

[8] K.B. Oldham, J. Spanier, The Fractional Calculus, Academic Press, New York, 1974.

[9] I. Podlubny, Fractional Differential Equations, Academic Press, San Diego, California-USA, 1999.

[10] I. Podlubny, R. L. Magin, and I. Trymorush , "Niels Henrik Abel and the birth of fractional calculus". Fractional Calculus and Applied Analysis, (2017) 20 (5), 10681075. doi:10.1515/fca-2017-0057.

[11] B. Ross, The development of fractional calculus 1695-1900, Historia Mathematica. 4 (1977), 7589. doi:10.1016/0315-0860(77)90039-8.

[12] S.G. Samko, A.A. Kilbas, O.I. Marichev, Fractional Integrals and Derivatives, Theory and Applications, Gordon and Breach, Yverdon et alibi, 1993. 